\title{
First-principles study for the adsorption of segments of BPA-PC on $\alpha-\mathrm{Al}_{2} \mathrm{O}_{3}(0001)$
}

\author{
Janne Blomqvist and Petri Salo \\ Department of Applied Physics, School of Science, Aalto University, P.O. Box 11100, FI-00076 Aalto, Finland
}

\begin{abstract}
We have studied the adsorption of bisphenol-A-polycarbonate (BPA-PC) on the $\alpha-\mathrm{Al}_{2} \mathrm{O}_{3}(0001)$ surface using density-functional theory (DFT) with van der Waals (vdW) corrections. The BPA-PC polymer can be divided into its chemical fragments which are phenylene, carbonate and isopropylidene groups. We have calculated the adsorption energy and geometry of the BPA-PC segments that consist of two to three adjacent groups of the polymer. Our DFT results show that the adsorption is dominated by the vdW interaction. It is also important to include the interaction of nearest-neighbor groups in order to provide a realistic environment for the adsorption of the polymer onto the surface. Our results also show that the BPA-PC molecule attaches to the alumina surface via the carbonate group located in the middle of the molecule chain
\end{abstract}

PACS numbers: 68.43.Fg, 68.47.Gh

The bisphenol-A-polycarbonate (BPA-PC) molecule studied in this work is an important industrial polymer used in different composite materials together with, e.g., metals. The molecule has extensively been studied both experimentally ${ }^{1-6}$ and computationally ${ }^{6-11}$. The interaction of BPA-PC or its chemical groups, phenylene, carbonate, and isopropylidene, with crystal surfaces has also been studied, and they provide a good model system for studying the properties of surfaces and interfaces ${ }^{8,11-18}$.

Density-functional theory (DFT) has become an important tool for the study of matter at the electronic-structure level, including processes on surfaces such as the adsorption of atoms or molecules. Even though the covalent interaction is crucial for the intramolecular binding or the binding of single atoms on surfaces, large molecules with closed electron shells are interacting relatively weakly with the surfaces. Large, especially organic, molecules interact with the surface mostly via the van der Waals $(\mathrm{vdW})$ interaction. This electric dipole-dipole interaction has a longer range compared to the covalent interaction, allowing the molecule to interact with the surface comparatively far from it ${ }^{19,20}$. However, the conventional DFT includes only the covalent interactions and thus it fails to give the correct adsorption energy of large molecules on surfaces. Recent developments have made it possible to include the effects of vdW interactions in the DFT description of the large molecule systems.

In this work we extend a subset of our previous DFT calculations for the groups of BPA-PC (Ref. ${ }^{12}$ ) by taking into account the interactions of nearest-neighbor groups as well as the vdW interactions. Both of these factors can be crucial in understanding the adsorption processes of large molecules, with macromolecules (polymers) being an extreme example. To show this, we have calculated the adsorption energy and geometry of different segments consisting of two to three adjacent groups of the BPA-PC polymer chain on the $\alpha$-alumina (0001) surface.

In the numerical calculations we combine two different DFT approaches depending on the purpose described below. Compared to adsorption calculations for small molecules or individual atoms, adsorption calculations for large molecules offer a number of challenges. First, in or- der to fit the molecule into the simulation box such that it does not interact with its own mirror image due to periodic boundary conditions, the box has to be large. Second, large molecules, especially segments of polymers studied here, have large conformational degrees of freedom, meaning that relaxing them requires a lot of time. These two issues, combined with the fact that one needs a similar level of accuracy as for normal adsorption calculations, essentially makes a traditional plane-wave DFT approach unfeasible. In order to perform these calculations, we have to make quick scans with a relative fast linear combination of atomic orbitals (LCAO) DFT method first and, after that, refining the interesting cases with a more accurate but significantly more expensive real-space finitedifference DFT method. This combining of two codes is significantly easier by controlling both of them via a unified high-level interface ${ }^{21}$.

For the LCAO calculations, we used the Siesta code ${ }^{22}$, using single- $\zeta$ polarized (SZP) orbitals and a single kpoint at the $\Gamma$ point. The many-body effects were approximated with the revised version ${ }^{23}$ of the Perdew-BurkeErnzerhof $^{24,25}$ (RPBE) form of the generalized-gradient approximation (GGA). The atomic coordinates were relaxed until the maximum force was less than $0.04 \mathrm{eV} / \AA$. The finite-difference calculations were done using projector augmented waves (PAW) ${ }^{26}$ as implemented in the real-space finite-difference code $\mathrm{GPAW}^{21,27,28}$. We used a grid spacing of approximately $0.19 \AA$, and a $2 \times 1 \times 1 \mathrm{k}$ point mesh using the Monkhorst-Pack scheme ${ }^{29,30}$. The exchange-correlation and vdW interactions were taken into account with a revised version of the PBE GGA GA,25,31 $^{24}$ (revPBE), and a self-consistent implementation of a fast Fourier transform (FFT) based method ${ }^{32}$ based on the vdW-functional of Dion et al. ${ }^{33,34}$. The systems were relaxed until the maximum force on all atoms were less than $0.02 \mathrm{eV} / \mathrm{A}$. The Fermi level was smeared using Fermi smearing with a smearing parameter of $0.1 \mathrm{eV}$.

The $\alpha-\mathrm{Al}_{2} \mathrm{O}_{3}(0001)$ supercell contained $96 \mathrm{Al}$ and $144 \mathrm{O}$ atoms in $12 \mathrm{Al}$ and $6 \mathrm{O}$ layers forming an $8 \times 4$ hexagonal surface supercell with Cartesian dimensions 8.3 $\AA$ x 19.1 $\AA$ x $27.0 \AA$. The height of the slab was $12.1 \AA$, and there was a $14.9 \AA$ vacuum region. The lowest $\mathrm{Al}$ and $\mathrm{O}$ layers 


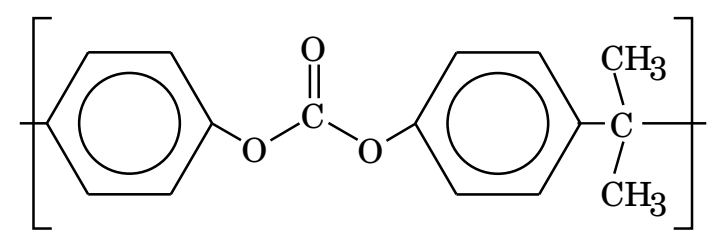

Figure 1. Structural formula of the BPA-PC monomer. The monomer consists of the groups P, C, P, and I, respectively.

were fixed, while the rest of the slab was relaxed. The surface was Al-terminated, as previous calculations have shown this to be the most stable termination ${ }^{35}$, although there are some conflicting experimental results suggesting a preference for a mixture of different terminations ${ }^{36}$. For some calculations, depending on the adsorbate configuration on the surface, the cell size was doubled to $16.6 \AA$ x $19.1 \AA$ x $27.0 \AA$.

A BPA-PC monomer can be seen in Fig. 1. The monomer consists of three different chemical groups, phenylene (marked with $\mathrm{P}$, chemical formula $\mathrm{C}_{6} \mathrm{H}_{4}$ ), carbonate $\left(\mathrm{C}, \mathrm{CO}_{3}\right)$, and isopropylidene $\left(\mathrm{I}, \mathrm{C}_{3} \mathrm{H}_{6}\right)$. As the BPA-PC polymer chains are usually terminated at both ends by a $\mathrm{P}$ group ${ }^{11}$, for the adsorption a PC segment was simulated. The adsorption of the PI segment has not been simulated, as the I group sterically prevents the P group from getting close to the surface. An IC segment has not been studied either, because it does not contain any $\mathrm{P}$ group. For the intra-chain adsorptions, segments consisting of three groups were simulated, but the chains of length of four groups or longer have not been simulated because of too time-consuming calculations. Thus, the segments studied are PC, PCP, IPC, and PIP. The dangling bonds of the neighbor groups have been passivated with $\mathrm{H}$ atoms. If single groups are simulated, the groups will be benzene $\left(\mathrm{C}_{6} \mathrm{H}_{6}\right)$, carbonic acid $\left(\mathrm{H}_{2} \mathrm{CO}_{3}\right)$, and propane $\left(\mathrm{C}_{3} \mathrm{H}_{8}\right)$ due to the $\mathrm{H}$ passivation.

First the minimum-energy adsorption configurations for each segment PC, PCP, IPC, and PIP on the $\alpha$ $\mathrm{Al}_{2} \mathrm{O}_{3}(0001)$ surface were searched for. The different adsorption sites used, the $\mathrm{Al}$ top, $\mathrm{Al}$ hollow, $\mathrm{O}$ top and $\mathrm{O}$ hollow sites, can be seen in Fig. 2. The adsorption site for a large molecule was determined so that we picked from the molecule an atom which we located at the adsorption site in each calculation. For PC and IPC the atom at the adsorption site was one of the $\mathrm{C}$ atoms in the $\mathrm{P}$ group and for PCP the atom was the $\mathrm{O}$ atom of the $\mathrm{C}$ group closest to the surface. For PIP there were two possible configurations, either one of the $\mathrm{C}$ or $\mathrm{H}$ atoms in the I group has been placed at the adsorption site. Furthermore, the rotation of the segment was chosen keeping in mind the steric constraints that a BPA-PC polymer chain could have had. Each segment was rotated at $30^{\circ}$ intervals, resulting in a total of 240 configurations. Symmetrically non-equivalent configurations were evaluated with the Siesta code using the fast LCAO method.

The Siesta results are quite qualitative and the differences between different configurations of the same system

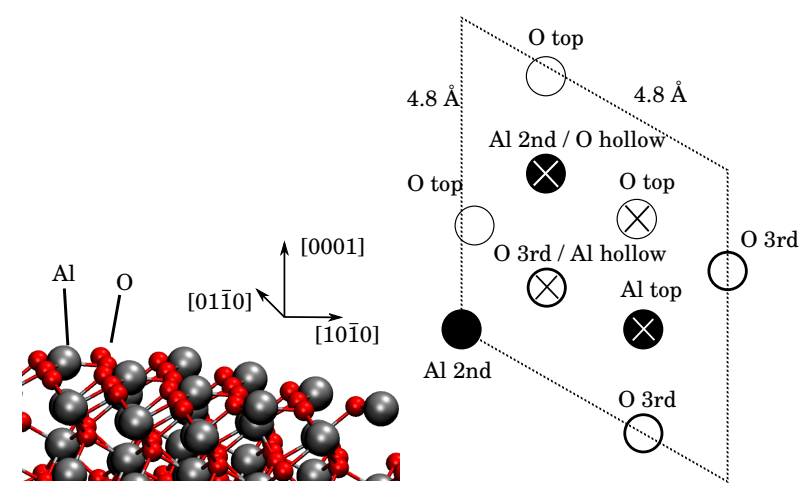

Figure 2. General view (left) and surface atoms (right) on the Al-terminated $\alpha-\mathrm{Al}_{2} \mathrm{O}_{3}(0001)$ surface. On the left, $\mathrm{Al}$ atoms are larger gray spheres and $\mathrm{O}$ atoms smaller red spheres (dark gray spheres in $\mathrm{b} / \mathrm{w}$ ). On the right, $\mathrm{Al}$ atoms are filled circles and $\mathrm{O}$ atoms empty circles. Due to the $\mathrm{Al}_{2} \mathrm{O}_{3}$ surface relaxation the uppermost $\mathrm{Al}$ and $\mathrm{O}$ layer are almost at the same height, then the second layer with $\mathrm{Al}$ atoms, and finally the third layer with $\mathrm{O}$ atoms. A 2x2 surface supercell box can also be seen.

Table I. Calculated adsorption energies with PAW vdW. The first column refers to the adsorption energy using self-consistent vdW DFT, the second column is the adsorption energy without the vdW contribution, and the third column is the vdW contribution to the adsorption energy.

\begin{tabular}{c|ccc}
\hline & \multicolumn{4}{|c}{$E_{\text {ads }}(\mathrm{eV})$} & $E_{\text {ads,revPBE }}(\mathrm{eV})$ & $E_{\mathrm{vdw}}(\mathrm{eV})$ \\
\hline PC & 1.22 & 0.42 & 0.80 \\
PCP & 1.49 & 0.22 & 1.26 \\
IPC & 1.47 & 0.32 & 1.15 \\
PIP & 0.90 & 0.03 & 0.88 \\
\hline
\end{tabular}

are some tenths of an electronvolt. This is to be expected since the large size of the segments means that they will not fit into a hollow site like an atom or a smaller molecule can do, and it also means that the notion of an adsorption site is at best approximate as the molecule segment interacts with the surface over a large area. The minimum adsorption configuration of the segment for each adsorption site is the O hollow site for PC and PCP, and the $\mathrm{O}$ top site for IPC and PIP with the $\mathrm{C}$ atom at the adsorption site. These systems have been studied further with more accurate, but time-consuming, finite-difference PAW scheme, including vdW interactions.

The results of the GPAW calculations using the revPBE-vdW functional are shown in Table I. One can see that the vdW contribution is very important, and especially for the adsorption of PIP on the alumina surface there is, in practice, no covalent interaction at all. The adsorption energies excluding vdW interactions are, at most, around half of the vdW contribution, confirming the hypothesis that including vdW interactions is essential for calculating large molecules on surfaces. In Fig. 3 one can see the adsorption geometries for the different segments on the surface.

One can see that the segments that contain the $\mathrm{C}$ group 


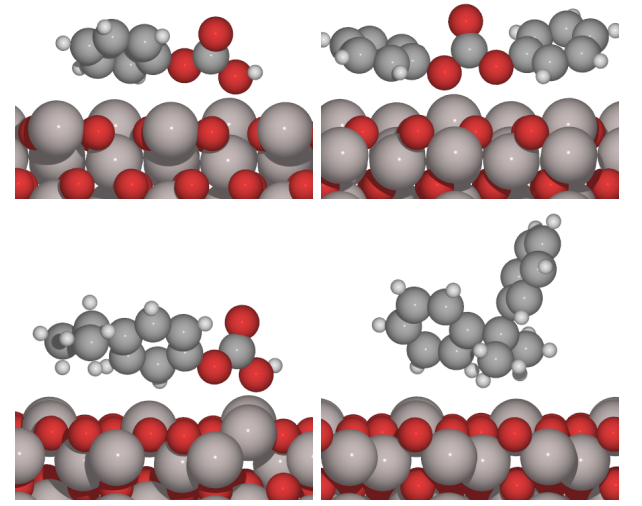

Figure 3. Equilibrium adsorption configurations for the segments on the surface. The segments are PC (top left), PCP (top right), IPC (bottom left), and PIP (bottom right). Red spheres (dark gray spheres in $\mathrm{b} / \mathrm{w}$ ) represent $\mathrm{O}$ atoms, large gray spheres $\mathrm{Al}$ atoms, gray spheres $\mathrm{C}$ atoms, and small gray spheres represent $\mathrm{H}$ atoms.

have larger adsorption energies (see Table I). The C group binds to the surface more eagerly than the other parts that can also be seen in Fig. 3. The surface $\mathrm{Al}$ atom underneath the $\mathrm{O}$ atom in the $\mathrm{C}$ group in each case has been pulled upward by about $0.2 \AA$ compared to the clean surface. This result is also consistent with the results in Refs. ${ }^{12,13}$ where it was found that phenol $\left(\mathrm{C}_{6} \mathrm{H}_{5} \mathrm{OH}\right)$ and carbonic acid binds to the $\mathrm{Al}_{2} \mathrm{O}_{3}$ surface via the oxygen atom in the phenol group and one of the oxygen atoms in the carbonic-acid group.

The neighboring groups are also important. The adsorption energy of the PC segment increases when the molecule chain contains an extra P forming a PCP segment on the surface. However, the increase is almost the same if one looks at the change of the adsorption energy between PC and IPC segments. As the PCP segment, the IPC segment interacts with the alumina surface via the $C$ group. The $\mathrm{P}$ group seems to be inert to the alumina surface, which is consistent with the results in Ref. ${ }^{12}$ where it was found that the benzene group does not bind to the alumina surface. In this work, the PIP segment that does not contain the $\mathrm{C}$ group interacts with the alumina surface via the I group and the $\mathrm{P}$ groups are only bound to the I group in the middle (see Fig. 3). However, if the chain contains four groups or more, it will contain at least one $\mathrm{C}$ group that will attach to the alumina surface. Unfortunately, the DFT calculations for chains with four groups or more become too time-consuming to perform. As the BPA-PC polymer chains are usually terminated at both ends by a $\mathrm{P}$ group ${ }^{11}$, we can conclude that this polymer chain attaches to the alumina surface via the $C$ group in the middle of the polymer.

The adsorption energy of these segments as a function of the perpendicular distance from the surface has also been calculated. For these calculations no geometry optimization was done, only the $z$ coordinate of all the atoms in the segment was changed. These results can be seen in

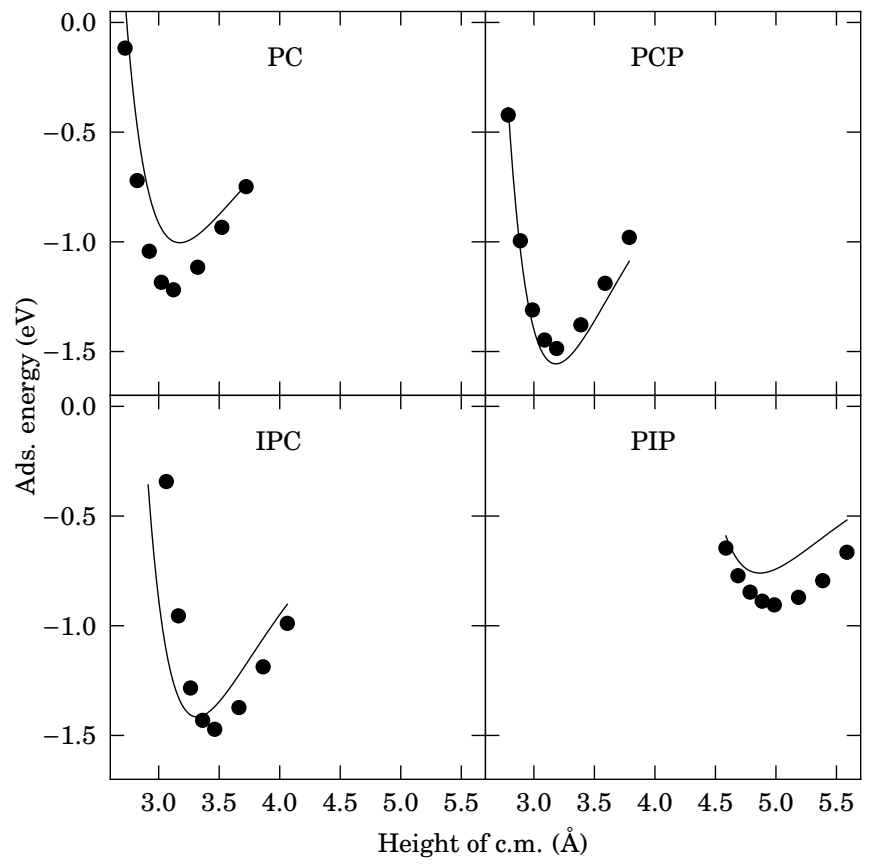

Figure 4. Adsorption energies as a function of the height of the segment center-of-masses from the surface. The solid line for each case represents an LJ fitting to the data.

Fig. 4, in which the adsorption energies of the segments are presented as a function of the height of the center of mass (c.m.) of the segment from the surface atomic layer. One can see that the equilibrium distance of the segment from the surface is pretty much the same, except for the PIP segment. This partly explains the smaller adsorption energy of PIP on the alumina surface.

We have also made a fit to the data in Fig. 4 using a 10-4 Lennard-Jones (LJ) potential

$$
E_{\mathrm{LJ}}^{10-4}(z)=\frac{5}{3} \epsilon\left[\frac{2}{5}\left(\frac{\sigma}{z}\right)^{10}-\left(\frac{\sigma}{z}\right)^{4}\right]
$$

which represents a 12-6 LJ potential integrated over the first atomic plane in the surface. In the fitting procedure, we have fitted sums of the single group potentials, that is, $\mathrm{P}+\mathrm{C}, \mathrm{P}+\mathrm{C}+\mathrm{P}, \mathrm{I}+\mathrm{P}+\mathrm{C}, \mathrm{P}+\mathrm{I}+\mathrm{P}$, and minimized, in the leastsquares sense, the difference between these sums and the vdW DFT segment data by varying the $\epsilon$ and $\sigma$ parameters for P, C, and I. The LJ fitting parameters are shown in Table II. For comparison, we have also listed in Table II the average adsorption energies and heights of single groups on the surface. Single groups were simulated in a fixed conformation as they were in the segments, and each of the dangling bonds of the groups was passivated with hydrogen atoms. These results also show that the adhesion of the $\mathrm{C}$ group to the alumina surface is the strongest. When one compares the LJ fitting parameter and the single-group results, the well depth $\epsilon$ of the LJ potential for the I group is not in line with the other results. This discrepancy can be understood if one looks at the PIP segment on the surfaces. The adhesion of PIP on 
Table II. Fitted LJ parameters and single group averaged vdW DFT results.

\begin{tabular}{l|cccc}
\hline & \multicolumn{1}{|c}{$(\mathrm{eV})$} & $\sigma(\AA)$ & $E_{\mathrm{ads}}(\mathrm{eV})$ & $z(\AA)$ \\
\hline $\mathrm{P}$ & 0.50 & 3.02 & 0.30 & 3.54 \\
$\mathrm{C}$ & 0.57 & 3.12 & 0.59 & 3.05 \\
$\mathrm{I}$ & 0.50 & 3.43 & 0.18 & 3.59 \\
\hline
\end{tabular}

the surface is mostly due to the I group and that is why the adsorption energy of a single I group in the fitting is so high.

The LJ fitting has also been shown in Fig. 4. The fitting seems reasonably good, considering the simplicity of the potential function, and the fitting is the same for all the cases. In particular, the fitting is good for the IPC and PCP segments, which are the most important cases, as PC is only at the chain end, and PIP is adsorbed far away from the surface. Since the LJ potential is a good approximation for the vdW interactions, the fitting confirms that the interaction between these segments and the alumina surface is mainly due to the vdW interactions, in agreement with Table I.
As a summary, we have calculated multi-group segments of the BPA-PC on an aluminum oxide surface using vdW DFT. The results show that, for these large segments, the vdW interaction plays a major role in the adhesion process at the atomic scale. Similarly, in order to provide a more realistic environment for each group on the surface, we have to include the nearest-neighbor groups, too. This is partly due to the long-range effect of the vdW interaction. We have also found that the BPA-PC molecule attaches to the alumina surface via the carbonate group located in the middle of the polymer chain. The LJ fittings of the interaction between the polymer fragments and the alumina surface can be used in moleculardynamics simulations in the future to study the properties of the interface between these materials.

This work has been supported by the Finnish Funding Agency for Technology and Innovation (Tekes) through the K3MAT project, and by the Academy of Finland through its Computational Nanoscience (COMP) Center of Excellence program headed by R. Nieminen. We also acknowledge the computer resources provided by the Finnish IT Center for Science (CSC). Finally, we wish to thank K. Johnston and A. Gulans for discussions.
1 J. Y. Jho and A. F. Yee, Macromolecules 24, 1905 (1991).

2 C. Kim and D. Paul, Macromolecules 25, 3097 (1992).

3 G. Floudas, T. Pakula, M. Stamm, and E. Fischer, Macromolecules 26, 1671 (1993).

4 G. Katana, F. Kremer, E. W. Fischer, and R. Plaetschke, Macromolecules 26, 3075 (1993).

5 U. Buchenau, C. Schönfeld, D. Richter, T. Kanaya, K. Kaji, and R. Wehrmann, Physical Review Letters 73, 2344 (1994).

6 J. Eilhard et al., The Journal of Chemical Physics 110, 1819 (1999).

7 C. F. Abrams and K. Kremer, Macromolecules 36, 260 (2003).

8 C. F. Abrams, L. Delle Site, and K. Kremer, Physical Review E 67, 021807 (2003).

9 J. Akola, P. Ballone, and R. O. Jones, Macromolecules 35, 2327 (2002).

10 J. Akola and R. O. Jones, Macromolecules 36, 1355 (2003).

11 L. Delle Site, C. F. Abrams, A. Alavi, and K. Kremer, Physical Review Letters 89, 156103 (2002).

12 J. Blomqvist and P. Salo, Journal of Physics: Condensed Matter 21, 225001 (2009).

13 S. D. Chakarova-Käck, Øyvind Borck, E. Schröder, and B. I. Lundqvist, Physical Review B 74, 155402 (2006).

14 L. Delle Site, A. Alavi, and C. F. Abrams, Physical Review B 67, 193406 (2003).

15 L. Delle Site, S. Leon, and K. Kremer, Journal of the American Chemical Society 126, 2944 (2004).

16 L. Delle Site, S. Leon, and K. Kremer, Journal of Physics: Condensed Matter 17, 53 (2005).

17 K. Johnston and R. M. Nieminen, Physical Review B 76, 085402 (2007).

18 K. Johnston, R. M. Nieminen, and K. Kremer, Soft Matter 7, 6457 (2011)

19 P. G. Moses, J. J. Mortensen, B. I. Lundqvist, and J. K.
Nørskov, The Journal of Chemical Physics 130, 104709 (2009).

20 J. Wellendorff et al., Topics in Catalysis 53, 378 (2010).

21 S. R. Bahn and K. W. Jacobsen, Computing in Science \& Engineering, Computing in Science \& Engineering 4, 56 (2002).

22 J. M. Soler et al., Journal of Physics: Condensed Matter 14, 2745 (2002)

23 B. Hammer, L. Hansen, and J. Nørskov, Physical Review B 59, 7413 (1999)

24 J. P. Perdew, K. Burke, and M. Ernzerhof, Physical Review Letters 77, 3865 (1996).

25 J. P. Perdew, K. Burke, and M. Ernzerhof, Physical Review Letters 78, 1396 (1997).

26 P. E. Blöchl, Physical Review B 50, 17953 (1994).

27 J. J. Mortensen, L. B. Hansen, and K. W. Jacobsen, Physical Review B 71, 035109 (2005).

28 J. Enkovaara et al., Journal of Physics: Condensed Matter 22, 253202 (2010).

29 H. J. Monkhorst and J. D. Pack, Physical Review B 13, 5188 (1976).

30 J. D. Pack and H. J. Monkhorst, Physical Review B 16, 1748 (1977).

31 Y. Zhang and W. Yang, Physical Review Letters 80, 890 (1998).

32 G. R. Pérez and J. M. Soler, Physical Review Letters 103, 096102 (2009).

33 M. Dion, H. Rydberg, E. Schröder, D. C. Langreth, and B. I. Lundqvist, Physical Review Letters 92, 246401 (2004).

34 M. Dion, H. Rydberg, E. Schröder, D. C. Langreth, and B. I. Lundqvist, Physical Review Letters 95, 109902 (2005).

35 J. Guo, D. E. Ellis, and D. J. Lam, Physical Review B 45, 13647 (1992).

36 J. Toofan and P. R. Watson, Surface Science 401, 162 (1998). 